\title{
Control of Milk Fermentation in Batch Bioreactor
}

\author{
Jozef Ritonja ${ }^{1, ~ *, ~ A n d r e j a ~ G o r s e k ~}{ }^{2}$, Darja Pecar ${ }^{2}$ \\ ${ }^{1}$ Faculty of Electrical Engineering and Computer Science, University of Maribor, \\ Koroska cesta 46, 2000 Maribor, Slovenia \\ ${ }^{2}$ Faculty of Chemistry and Chemical Engineering, University of Maribor, \\ Smetanova ulica 17, 2000 Maribor, Slovenia \\ jozef.ritonja@um.si
}

\begin{abstract}
In this paper, modelling and control of a batch bioreactor is studied. A main disadvantage of batch bioreactors compared to other types of bioreactors is their inability to introduce biological or/and chemical substances during operation. Therefore, possibility of bioreactor's control by means of changing temperature was proposed, analyzed, and implemented. A new supplementary input/output dynamical mathematical model, which considers influence of heating and cooling on a bioprocess, was developed. On a basis of this model, a control system was designed and a method for tuning of the controller was suggested. Results show characteristics, applicability, and advantages of the presented approach.
\end{abstract}

Index Terms-Batch bioreactor; Fermentation process; Modelling; Control.

\section{INTRODUCTION}

High demand for biologically manufactured products, such as food, beverages, specialty chemicals, and particularly pharmaceuticals, is the main reason for rapid evolution of biotechnology industry [1]. The heart of most biotechnological systems is the bioreactor, in which treated materials promote biotransformation by action of living cells or by cellular components, such as enzymes [2]. The bioreactor is a tank or a vessel, often cylindrical and made of stainless steel, where cells or cell-free enzymes transform raw materials into biochemical products and/or less undesirable by-products. This process unit can also be a device or a system for growth of cells or tissues in a context of a cell culture for the tissue or other applications involving biochemical engineering.

Based on a mode of operation, bioreactors may be classified as continuous, fed-batch, and batch. In the continuous operation mode, a substrate is continuously added and a product is continuously removed. The output from the continuous reactor can be altered by varying run time. This increases operating flexibility for manufacturers and makes the continuous operation more economic than others make. Main characteristics of the fed-batch bioreactor are feeding of a sterile substrate, absence of outflow from a fermenter, and increase of volume [3]. Required nutrients are also added continuously or intermittently to initial medium after start of cultivation. The fed-batch processes

Manuscript received 13 May, 2019; accepted 4 January, 2020 are used mainly to avoid utilizing substrates that inhibit a growth rate, if present at high concentrations [4].

Utilization of the batch processes has increased significantly nowadays. They are extensively used to produce special biomolecules for use in chemical, biotechnological, and pharmaceutical industries. Production of these high value-added bioproducts contributes to a significant and growing portion of revenue and earnings of the bioprocess industries [5]. In the batch processes, most of the materials required are loaded onto the bioreactor aseptically and removed at the end of the operation. Only materials added and removed during the operation are exchanging gas/air, antifoam, and $\mathrm{pH}$ controlling agents.

The bioreactor, in general, is any manufactured or engineered system that supports a biologically active environment. Controlling life and productivity of living organisms is a complex task, because of the requirement to obtain a specific target product, biomass, and/or metabolites. Each microorganism and target product have their critical state, where this product is produced at the maximum quantity and at desired quality [6]. Thus, an objective of the control in the bioprocess is to maximize production of a desired product. For the batch bioreactors, this means maximizing the quality of the desired product at the end of each batch.

For optimal production in bioreactors, the growth of microorganisms should be controlled. This is not difficult to achieve in the fed-batch and continuous bioreactors, where one or more substrates are supplied to the bioreactor during a reaction influencing the growth of microorganisms. It is found much more complicated to control the culture growth in batch bioreactors. They operate as closed systems, where addition of the substrates is not allowed during the cultivation. Therefore, bioprocesses in batch reactors are not feasible to control by means of supplying additional substrates into the reactor. Accordingly, the commercial batch bioreactors are not equipped with the control systems, which would insure the optimal dynamic growth of the microorganisms. Almost all batch bioreactors have a heating device with a control system, which enables to maintain the desired temperature of the fermentation. This heating system is relatively fast - its time constant is much shorter than the time constants of the bioprocesses, which means that temperature of the reaction media changes much faster than 
the microorganism's activity.

The main contribution of the proposed paper is the study of possibility of implementation of the heating system of the bioreactor as the actuator, which will realize a desired time response of the growth of the microorganism. In Section II, the dynamic mathematical model describing a correlation between the reaction media temperature and the growth of the microorganisms is described. The model is developed on the basis of thorough and systematic experimental work. In Section III, the system for the control of the growth of the microorganisms is designed on the basis of the heating system. A prosperous synthesis procedure found on a root locus diagram is developed and presented. Results are shown in the Section IV.

\section{Mathematical Model of the BAtCH Bioreactor}

\section{A. Description of the Batch Bioreactor}

The control of the fermentation process was studied in reaction calorimeter RC1e from Mettler Toledo. The RC1e is a computer-controlled laboratory batch reactor with working volume $0.7 \mathrm{~L}$, primarily designed for determination of thermal characteristics of chemical reactions. The used batch bioreactor was equipped with additional actuators and sensors, which enable identification and control of basic biochemical and physical quantities during the fermentation process. The bioreactor used is shown in Fig. 1.

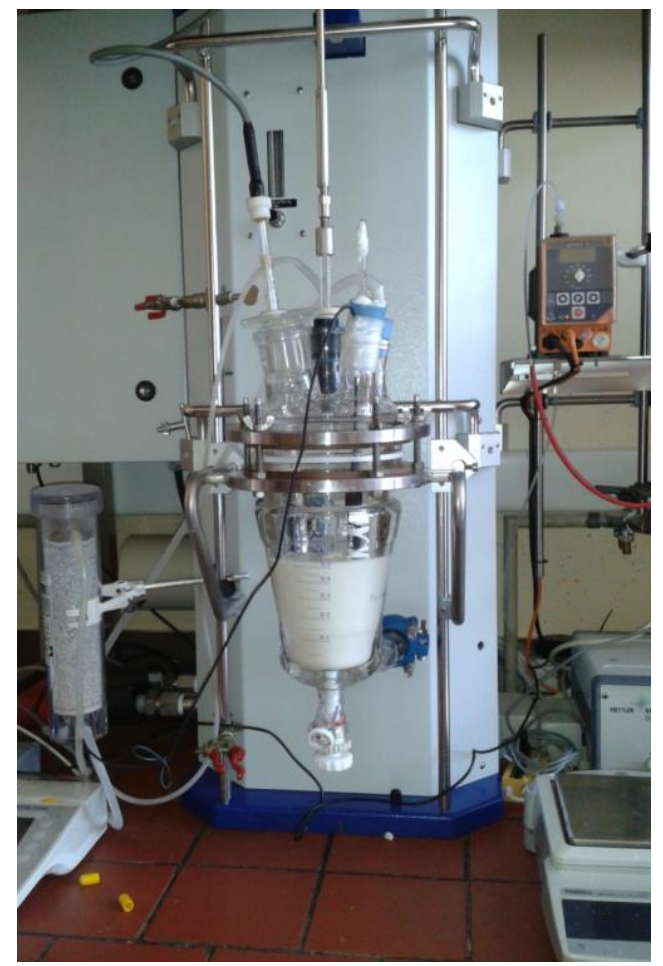

Fig. 1. Batch reactor RC1e from Mettler Toledo.

In our case, dissolved $\mathrm{CO}_{2}$ was chosen as an output and a controlled variable. The SevenMulti Apparatus (Mettler Toledo) equipped with an ISE51B ion selective electrode was used for monitoring dynamics of $\mathrm{CO}_{2}$ in liquid media. It is connected to a personal computer, which records measurements in a selected time period with Lab X direct $\mathrm{pH} 2.3$ software. The electrode has a selective permeable membrane, which separates the medium from the electrolyte in the electrode. Dissolved $\mathrm{CO}_{2}$ from the medium diffuses through the membrane until equilibrium between the partial pressure of $\mathrm{CO}_{2}$ in the electrolyte and the partial pressure of $\mathrm{CO}_{2}$ in the medium is reached. Since partial pressure is proportional to concentration, the concentration of $\mathrm{CO}_{2}$ in the medium can be determined from a calibration curve.

\section{B. Autonomous Mathematical Model}

A typical cells growth curve in the batch fermenter consists of lag time or an induction phase followed by an exponential growth phase, a stationary phase, and, in the end, a dying phase. Cells use the lag phase to adapt to their new environment. Following the lag period, the growth starts in the acceleration phase and continues through the growth and decline phases. If the growth is exponential, the growth phase resembles a straight line. As nutrients in the culture medium become depleted or inhibitory products accumulate, the growth slows down and the cells enter the decline phase. After this transition period, the stationary phase is reached, during which no further growth occurs. Some cultures exhibit a death phase as the cells lose viability due to the lack of food.

There are numerous models of fermentation processes based on different kinetic assumptions. Most of them focus only on the process kinetics; some are more complicated and involve equations for heat transfer, the temperature dependence of kinetic parameters, oxygen mass transfer. The classical mathematical models are mainly built of the mass balances of the main components involved in the culture (biomass, substrates, metabolites, and products). They describe global behavior of a given component or an effect of some biochemical reactions involving the component. A concept of a specific growth rate is often used. It consists of modelling various influences of the growth kinetics. Substrate limitation is included in a Monod's and Tessier's law, substrate limitation and inhibition are considered in a Haldane's law, substrate limitation and biomass inhibition are defined in a Contois' law, etc. [1].

The consumption or production of major components during the milk fermentation process in the batch bioreactor can be expressed by a set of nonlinear differential equations $[1],[7]$ :

$$
\begin{gathered}
\dot{X}=\mu(S, P) X, \\
\dot{S}=-\mu(S, P) X, \\
\dot{P}=[\alpha \mu(S, P)+\beta] X,
\end{gathered}
$$

where $X$ is the concentration of the cellular biomass, $S$ is the concentration of the growth limiting substrate (e.g., glucose), $P$ is the concentration of the desired product (e.g., ethanol), $\mu(S, P)$ is a specific growth rate, parameter $\alpha$ is inverse of the product yield associated with the cellular growth, and parameter $\beta$ is inverse of the growthindependent product yield. Although yield coefficients $\alpha$ and $\beta$ often vary with the environmental conditions, the both coefficients are usually treated as constant. The overall accuracy of the bioreactor model depends strongly on identification of a growth-rate function that adequately describes the cellular growth over the range of environmental conditions. The function is expressed as 


$$
\mu(S, P)=\frac{\mu_{\mathrm{m}}\left(1-\frac{P}{P_{\mathrm{m}}}\right) S}{K_{\mathrm{m}}+S+\frac{S^{2}}{K_{\mathrm{i}}}},
$$

where $\mu_{\mathrm{m}}$ is the maximum growth rate, $K_{\mathrm{m}}$ is the substrate saturation constant, $K_{\mathrm{i}}$ is the substrate inhibition constant, and $P_{\mathrm{m}}$ is the product inhibition constant, which is sufficiently general and accurate enough to describe many situations of practical interest [1]. In many situations, instead of (4), a simplified rational expression called a Monod's law also is utilized as follows

$$
\mu(S, P)=\frac{\mu_{\mathrm{m}} S}{K_{\mathrm{m}}+S} .
$$

In this case, the specific growth rate depends only on substrate concentration.

The development and the analysis of the mathematical model were established for the described batch reactor and milk fermentation with kefir grains [8]. The $\mathrm{CO}_{2}$ was selected as the output product of the bioprocess. The time response of $\mathrm{CO}_{2}$ during the fermentation process without the control system is shown in Fig. 2. In this case, the reactor's media temperature $\left(T=22^{\circ} \mathrm{C}\right)$ stays constant during the entire fermentation process.

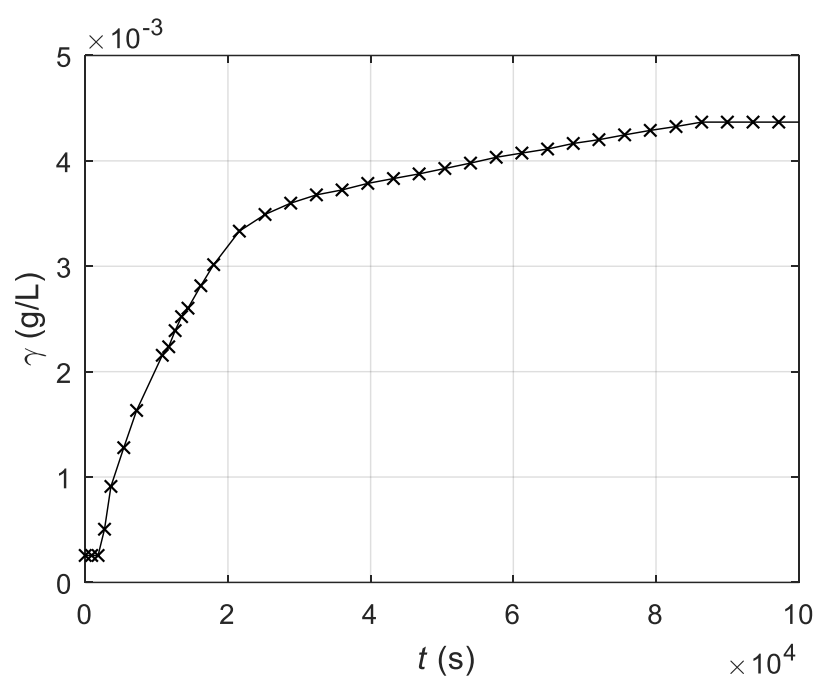

Fig. 2. Time response of $\mathrm{CO}_{2}$ concentration $\gamma$ for non-controlled batch reactor by constant reactor's media temperature $\left(T=22^{\circ} \mathrm{C}\right)$.

The obtained $\mathrm{CO}_{2}$ time response is similar to the step response of the first order differential equations. This is consistent with the known mathematical model of the noncontrolled fermentation [8]. Deviations from the response of the first order differential equations come from nature of the biochemical process itself. Activity of microorganisms is often varying, since they are highly sensitive to changes (temperature, concentration, and $\mathrm{pH}$ ) of process conditions, changes of their viability, and potential mutations. The disturbances are clearly seen in the non-linear model of the fermentation process also.

\section{Supplementary Input/Output Mathematical Model}

Almost all known mathematical models of the batch bioreactors (also the presented one, equations (1)-(5)) represent a bioreactor as an autonomous system. They describe only the time responses of the bioreactor's media subject to the different initial conditions. These models are not applicable for control system design, because they do not express the influence of the control variables on the major bioreactor's variables: biomass, a substrate, and product concentration.

For the purpose of design of the batch bioreactor's control system, a mathematical model connecting a controllable input variable (heat) and bioreactor's media variables is required. There are only few publications available wherein the influence of the heat (reactor's media temperature) on the state variables is studied [8].

Within a domain of our research, we tried to analyze the influence of the heat on the growth of the microorganisms experimentally. We tested the bioreactor's response on different heat's variations in different phases of the fermentation process. The investigated bioreactor's output variable was $\mathrm{CO}_{2}$.

Our goal was to develop a mathematical model that would satisfactorily describe the dynamics of the fermentation process in a batch bioreactor while keeping the mathematical model as simple as possible in its underlying methodology of the parameter determination and its presented form. The obtained mathematical model must also be suitable for the design of the control system of the batch bioreactor.

Therefore, to obtain the appropriate model, we tested a range of specific sub-models with various connections. The main conclusion of the comprehensive laboratory work allows us to consider the bioreactor's mathematical model composed of two parts:

- An autonomous mathematical model, which describes dynamics of the bioreactor by constant media temperature;

- A supplementary input/output mathematical model, which describes the influence of heat on the $\mathrm{CO}_{2}$ release.

The autonomous model describes the dynamics of the fermentation process in absence of changes in input control quantities. This model is important for determination of the reference trajectory of the output variable. The autonomous model could be derived from the time response of $\mathrm{CO}_{2}$ concentration when no change of temperature of the reactor's media occurs.

The supplementary input/output mathematical model is required for the design and synthesis of the control system of the batch bioreactor. It could be obtained from the comparison of the time response of $\mathrm{CO}_{2}$ concentration for constant temperature of the reactor's media and the time response of $\mathrm{CO}_{2}$ concentration subject to a step change of temperature of the reactor's media. Figure 3 shows the influence of the step change of the bioreactor's media temperature on the $\mathrm{CO}_{2}$ release during the operation. It presents the trajectory of the bioreactor's output $\left(\mathrm{CO}_{2}\right)$ when the bioreactor's media temperature is constant during the operation $\left(T=22{ }^{\circ} \mathrm{C}\right)$ and the time response of $\mathrm{CO}_{2}$ on the step change of the reactor's media temperature from $T=$ $22{ }^{\circ} \mathrm{C}$ to $T=27{ }^{\circ} \mathrm{C}$. The step change occurs at $t=10,800 \mathrm{~s}$. The supplementary input/output mathematical model represents the connection between a step change of the temperature of the reactor's media and supplementary 
production of $\mathrm{CO}_{2}$.

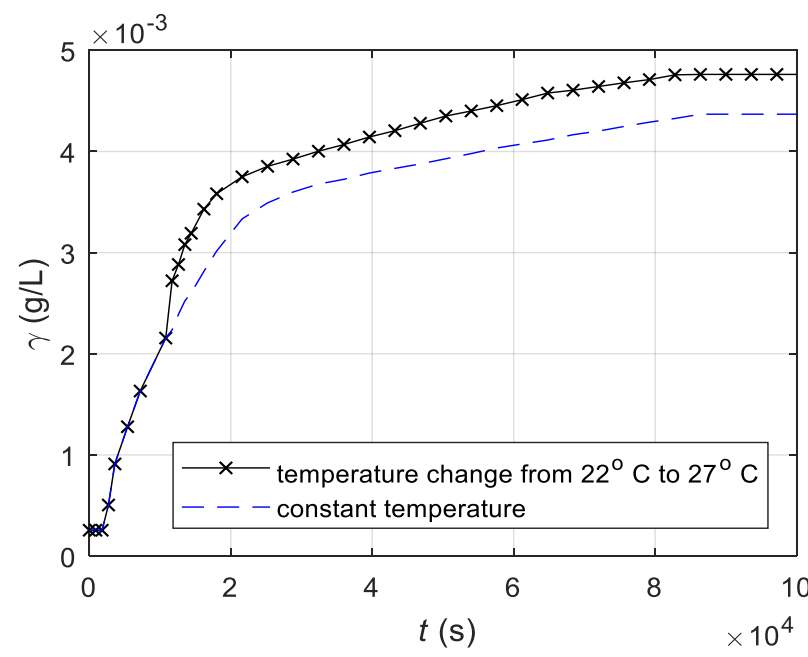

Fig. 3. a) Time response of $\mathrm{CO}_{2}$ concentration $\gamma$ when step change of reactor's media temperature from $T=22{ }^{\circ} \mathrm{C}$ to $T=27{ }^{\circ} \mathrm{C}$ occurs at $t=$ $10,800 \mathrm{~s}$ (solid line); b) Time response of $\mathrm{CO}_{2}$ concentration $\gamma$ for constant reactor's media temperature $\left(T=22{ }^{\circ} \mathrm{C}\right)$ (dashed line).

From the analysis of the results of the laboratory experiments, it became noticeable that supplementary input/output mathematical model must describe the behavior of the bioreactor during the steady state operation and during the transient response. Based on the many measured responses, we tested different structures of the model. For the described bioreactor and the studied fermentation process, we discovered that this model could be presented as a dynamical system built of two parallel-connected processes:

- A first order term with gain $k_{1}$ and time constant $T_{1}$;

- A second order term with gain $k_{2}$, time constant $T_{2}$, and damping $z$, which is in series with differentiator with time constant $T_{\mathrm{d}}$.

The first order term denotes the major (dominant) connection between the bioreactor's temperature and the dynamics of $\mathrm{CO}_{2}$ release. The second part (the second order term with the differentiator) has an impact during the transient response and is important for tuning of the controller.

The proposed supplementary input/output mathematical model is linear and non-autonomous. To obtain the parameters of the supplementary input/output mathematical model, a least square identification method [8] was utilized. The influence of the temperature changes on the parameters is not considerable.

The differences between the trajectory of the bioreactor's output $\left(\mathrm{CO}_{2}\right)$ by constant temperature during the entire operation and the time responses of the $\mathrm{CO}_{2}$ release affected by different changes of the reactor's media temperature were used to identify the supplementary input/output mathematical model. For the described bioreactor and the studied fermentation process, the following parameters of the supplementary input/output model were calculated and presented in Table I.

TABLE I. THE PARAMETERS OF THE SUPPLEMENTARY INPUT/OUTPUT MODEL.

\begin{tabular}{|c|c|c|c|}
\hline$k_{1}=7.87 \times 10^{-5}$ & $T_{1}=800 \mathrm{~s}$ & & \\
\hline$k_{2}=1.8$ & $T_{2}=5000 \mathrm{~s}$ & $z=0.36$ & $T_{\mathrm{d}}=0.2 \mathrm{~s}$ \\
\hline
\end{tabular}

Figure 4 shows the fitting of the measured results of the fermentation process of the batch bioreactor with the simulation results obtained from the identified mathematical model. To obtain a measured difference, two experiments involving a realistic process were conducted. One experiment was carried out with constant temperature during the entire operation and other experiment was carried out by a step change of the temperature of the reactor's media from $T=22^{\circ} \mathrm{C}$ to $T=27^{\circ} \mathrm{C}$ at time $t=10,800 \mathrm{~s}$ (shown in Fig. 3). The identified difference was calculated from the obtained supplementary input/output model. The integral square error quality criterion was used to confirm the fitting of the response of the identified model with the measured trajectory.

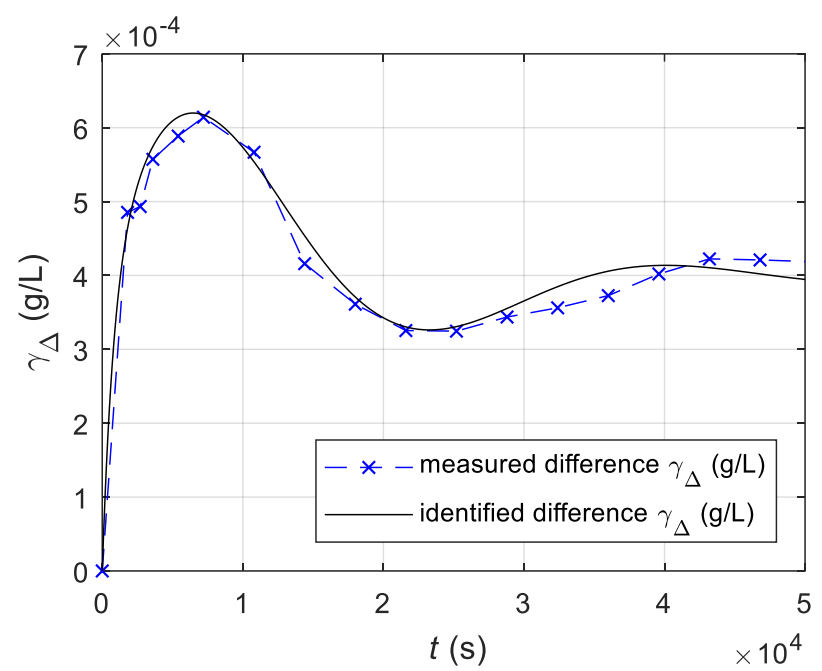

Fig. 4. Measured and identified time response of supplementary input/output model, which appears from step change in temperature from $T$ $=22{ }^{\circ} \mathrm{C}$ to $T=27^{\circ} \mathrm{C}$.

The block diagram of the mathematical model of the batch bioreactor is presented in Fig. 5 .

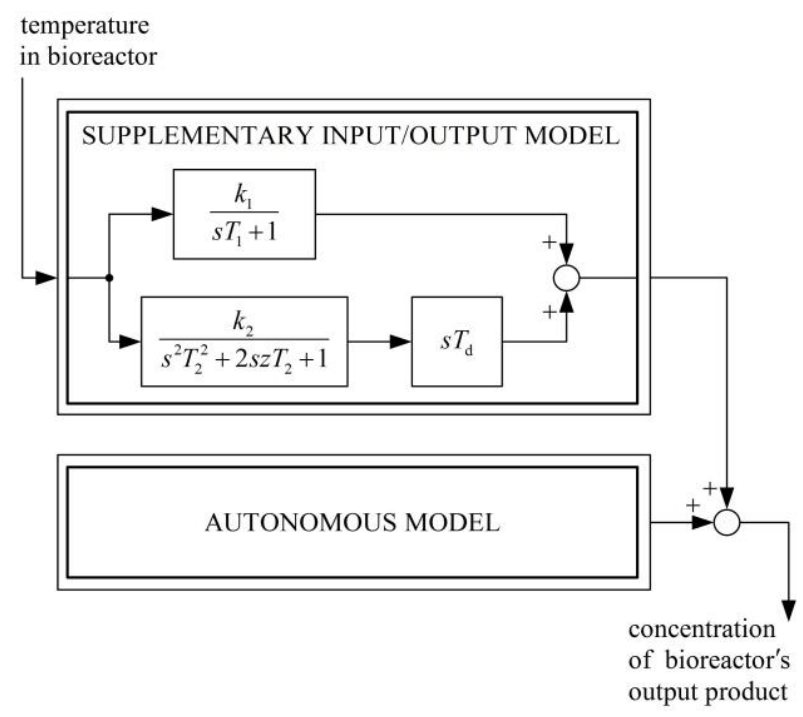

Fig. 5. Block diagram of mathematical model of batch bioreactor.

\section{CONTROL System OF THE BATCH BIOREACTOR}

\section{A. Control System Structure}

The aim of the research was to show the applicability of the bioreactor's heating system for the development of control system, which will realize the controlled growth of 
the microorganisms. The proposed control system consists of three parts:

- The measurement system, which enables on-line measurement of the $\mathrm{CO}_{2}$ concentration;

- The system to calculate the reference time trajectory of the $\mathrm{CO}_{2}$ concentration;

- The controller.

The block diagram of the entire control system is shown in Fig. 6.

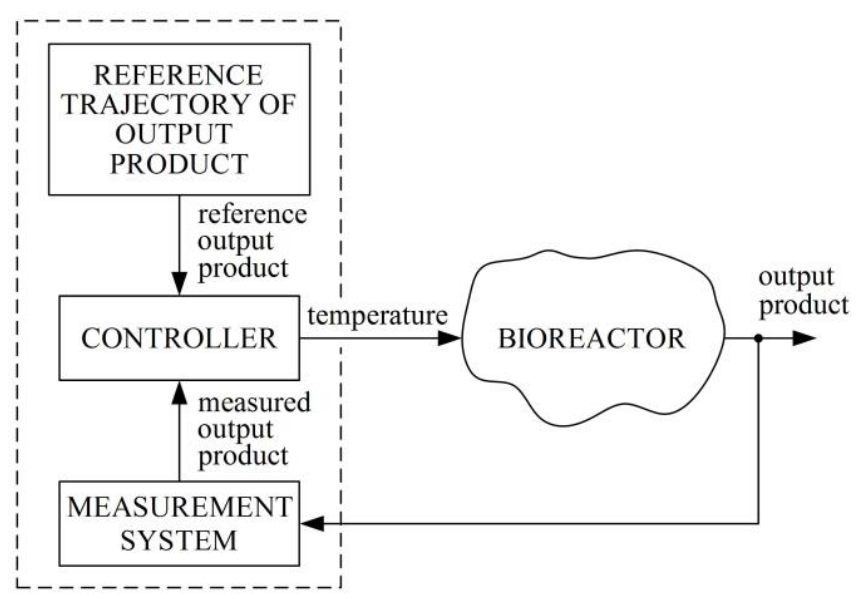

Fig. 6. Block diagram of system for $\mathrm{CO}_{2}$ release control.

\section{B. Control System Synthesis}

The PI (Proportional Integral) controller was used for control implementation. Its parameters are calculated from the supplementary input/output mathematical model parameters by means of a root locus diagram. Good results were obtained by use of the simple two-stage procedure [9], [10]:

1. Integral time constant $T_{\mathrm{i}}$ of the PI controller is calculated in regards to time constant $T_{1}$ in order to fulfil condition $T_{\mathrm{i}}<T_{\mathrm{i}}$;

2. PI controller gain $k$ was chosen from the root locus diagram in such a way that the four dominant closed loop poles will be sufficiently damped. The root locus diagram of the studied system is shown in Fig. 7.

For the analyzed bioreactor, the following values of the PI controller were calculated: $k=2000, T_{\mathrm{i}}=100 \mathrm{~s}$.

\section{RESULTS}

The developed control system was used for the control of the $\mathrm{CO}_{2}$ release of the batch bioreactor. The output product trajectory must assure economical production of the high quality product. To obtain such output trajectory, the appropriate reference output trajectory must be generated. The reference output trajectory was determined on a basis of empirical knowledge of the analyzed fermentation process. For the studied fermentation process, the reference trajectory of the $\mathrm{CO}_{2}$ concentration was obtained by means of slight modification of the response of the autonomous model. Compared to the response of the autonomous model, the reference trajectory has a smaller gradient in the beginning phase of the fermentation process $(t<5,000 \mathrm{~s})$, quicker progression to the steady state value $(\mathrm{t} \approx 40,000 \mathrm{~s})$, and then little increase of the steady state value.

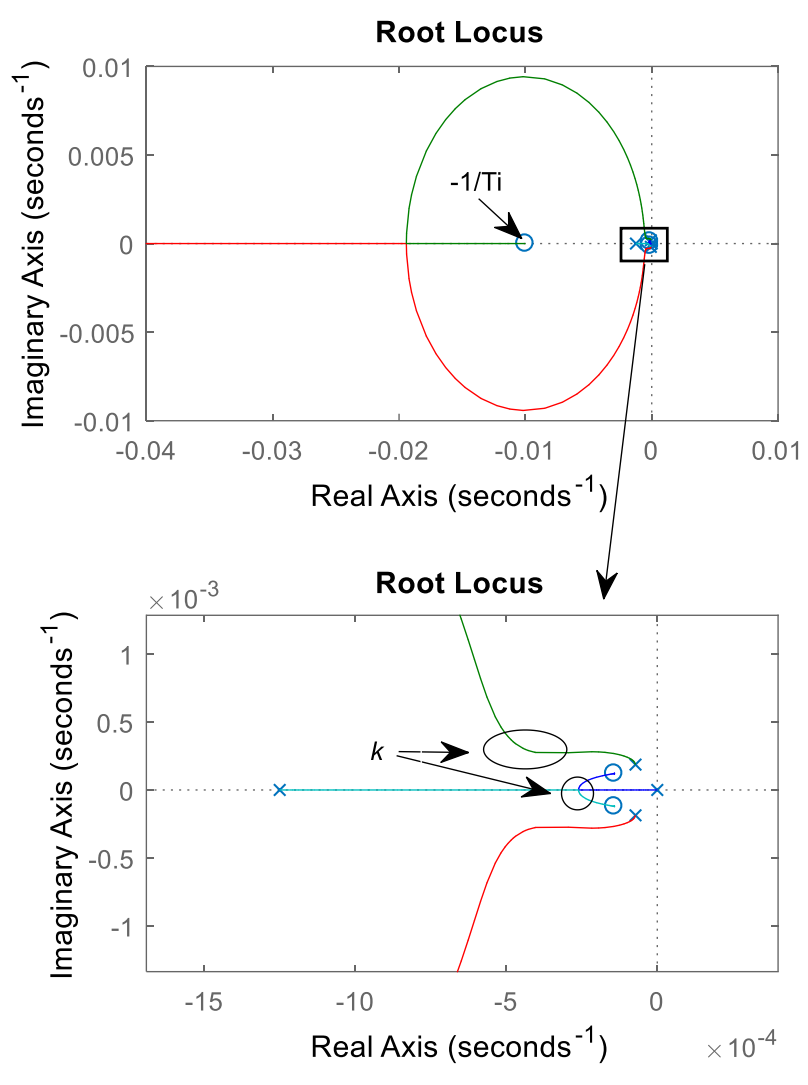

Fig. 7. Root locus diagram based on supplementary input/output model.

The determined reference output trajectory is described with (6), such as

$$
\begin{gathered}
\gamma_{r}(t)=4.75 \times 10^{-3}-6.42 \times 10^{-7} t e^{-1.43 \cdot 10^{-4} t}- \\
-4.49 \times 10^{-3} e^{-1.43 \cdot 10^{-4} t}
\end{gathered}
$$

Figure 8 shows the reference and measured trajectories of the $\mathrm{CO}_{2}$ concentration when the proposed control system for the batch bioreactor was used. The almost perfect following of the reference trajectory was achieved.

The necessary variation of the reactor's media temperature is shown in Fig. 9. It is seen that the temperature deviations to assure the desired control are not large.

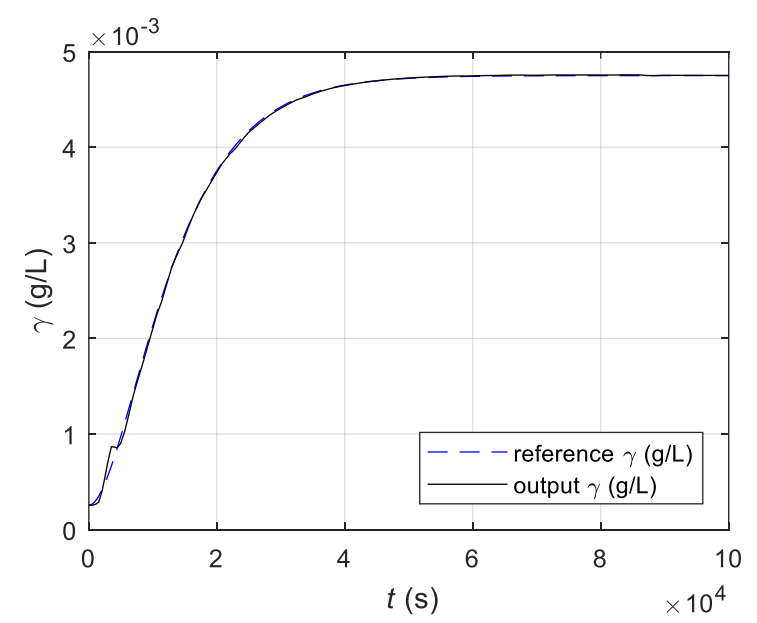

Fig. 8. Reference and measured trajectories of the $\mathrm{CO}_{2}$ concentration $\gamma$ when proposed control system for batch bioreactor was used. 


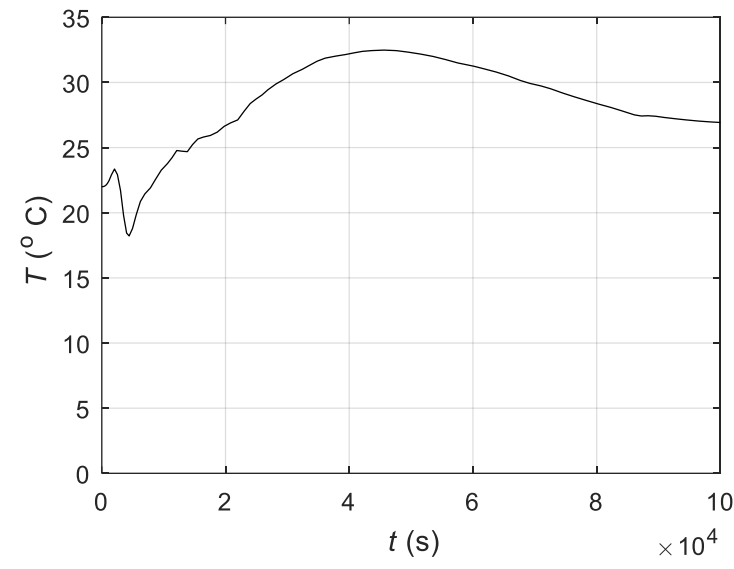

Fig. 9. Time response of reactor's media temperature $T$ for control of $\mathrm{CO}_{2}$ release.

\section{CONCLUSIONS}

The main conclusion of the paper is that we can ensure the controlled growth of microorganisms in batch bioreactors by means of a bioreactor's heating system, i. e., varying temperature of the process. This means that the growth of the microorganisms will follow the reference trajectory of the $\mathrm{CO}_{2}$ concentration being selected in such a way that the product of the fermentation will be of a high quality and will be produced in prescribed time and energy efficient also.

The addressed challenge is a part of contemporary issues that needs to be further investigated. Following the results of this paper, the operation of batch bioreactors could be significantly improved. We estimate that the advanced concept will be used in such commercial systems in the near future.

\section{CONFLICTS OF INTEREST}

The authors declare that they have no conflicts of interest.

\section{REFERENCES}

[1] M. A. Henson, "Biochemical reactor modeling and control", IEEE Control Systems Magazine, vol. 26, no. 4, pp. 54-62, Aug. 2006. DOI: 10.1109/MCS.2006.1657876.

[2] A. Pandey, C. Larroche, C. R. Soccol, and C. G. Dussap, "Chapter 7, Bioreactors: Functions in fermentation processes", Advances in Fermentation Technology. Asiatech Publishers Inc., New Delhi, 2008, pp. 172-201.

[3] I. J. Dunn, E. Heinzle, J. Ingham, and J. E. Prenosil, Biological Reaction Engineering: Dynamic Modelling Fundamentals with Simulation Examples. Wiley-VCH, Verlag, Weinhem, 2003. DOI: $10.1002 / 3527603050$.

[4] A. Nag, Biofuels Refining and Performance. McGraw-Hill Professional, 2008.

[5] A. Cinar, S. J. Parulekar, C. Ündey, and G. Birol, Batch Fermentation, Modeling, Monitoring, and Control. Marcel Dekker, New York, Basel, 2003. DOI: 10.1201/9780203911358.

[6] K. Yamuna Rani and V. S. Ramachandra Rao, "Control of fermenters - A review", Bioprocess Engineering, vol. 21, no. 1, pp. 77-88, Jul. 1999. DOI: 10.1007/PL00009066.

[7] G. Bastin and D. Dochain, On-Line Estimation and Adaptive Control of Bioreactors. Elsevier, 1990. DOI: 10.1016/B978-0-444-884305.50009-8.

[8] R. Isermann and M. Muenchhof, Identification of Dynamic Systems. Springer Verlag, Berlin, 2011.

[9] K. H. Ang, G. Chong, and Y. Li, "PID control system analysis, design and technology", IEEE Transactions on Control Systems Technology, vol. 13, no. 4, pp. 559-576, Jul. 2005. DOI: 10.1109/TCST.2005.847331.

[10] K. Astrom and T. Hagglund, PID Controllers: Theory, Design and Tuning. Instrument Society of America, 1995. 